Original paper

\title{
Measuring the dose in bone for spine stereotactic body radiotherapy
}

\author{
Maddison Shaw a,b, ", Jessica Lye ${ }^{\text {a,c }}$, Andrew Alves ${ }^{\text {a }}$, Maximilian Hanlon d, Joerg Lehmann e,f,g, h, \\ Jeremy Supple $^{\mathrm{a}}$, Claudiu Porumb ${ }^{\mathrm{i}}$, Ivan Williams ${ }^{\mathrm{j}}$, Moshi Geso ${ }^{\mathrm{b}}$, Rhonda Brown ${ }^{\mathrm{a}}$ \\ a Australian Clinical Dosimetry Service, Australian Radiation Protection and Nuclear Safety Agency, Melbourne, Australia \\ ${ }^{\mathrm{b}}$ School of Health and Biomedical Sciences, RMIT University, Melbourne, Australia \\ ${ }^{\mathrm{c}}$ Olivia Newton John Cancer Wellness Centre, Melbourne, Australia \\ ${ }^{\mathrm{d}}$ Primary Standards Dosimetry Laboratory, ARPANSA, Melbourne, Australia \\ ${ }^{\mathrm{e}}$ Department of Radiation Oncology, Calvary Mater Newcastle, Newcastle, Australia \\ ${ }^{\mathrm{f}}$ School of Science, RMIT University, Melbourne, Australia \\ ${ }^{g}$ School of Mathematical and Physical Sciences, University of Newcastle, Australia \\ ${ }^{\mathrm{h}}$ Institute of Medical Physics, University of Sydney, Australia \\ ${ }^{\mathrm{i}}$ Alfred Health Radiation Oncology, The Alfred Hospital, Melbourne, Australia \\ ${ }^{\mathrm{j}}$ Australian Radiation Protection and Nuclear Safety Agency, Melbourne, Australia
}

\section{A R T I C L E I N F O}

\section{Keywords:}

SBRT

Dosimetry audit

Dosimetry

Quality assurance

Dose to medium

\begin{abstract}
A B S T R A C T
Purpose: Current quality assurance of radiotherapy involving bony regions generally utilises homogeneous phantoms and dose calculations, ignoring the challenges of heterogeneities with dosimetry problems likely occurring around bone. Anthropomorphic phantoms with synthetic bony materials enable realistic end-to-end testing in clinical scenarios. This work reports on measurements and calculated corrections required to directly report dose in bony materials in the context of comprehensive end-to-end dosimetry audit measurements (63 plans, 6 planning systems).

Materials and methods: Radiochromic film and microDiamond measurements were performed in an anthropomorphic spine phantom containing bone equivalent materials. Medium dependent correction factors, $\mathrm{k}_{\text {med }}$, were established using $6 \mathrm{MV}$ and $10 \mathrm{MV}$ Linear Accelerator Monte Carlo simulations to account for the detectors being calibrated in water, but measuring in regions of bony material. Both cortical and trabecular bony material were investigated for verification of dose calculations in dose-to-medium $\left(D_{m, m}\right)$ and dose-to-water $\left(D_{w, w}\right)$ scenarios. Results: For $\mathrm{D}_{\mathrm{m}, \mathrm{m}}$ calculations, modelled correction factors for cortical and trabecular bone in film measurements, and for trabecular bone in microDiamond measurements were $0.875( \pm 0.1 \%), 0.953( \pm 0.3 \%)$ and $0.962( \pm 0.4 \%)$, respectively. For $\mathrm{D}_{\mathrm{w}, \mathrm{w}}$ calculations, the corrections were $0.920( \pm 0.1 \%), 0.982( \pm 0.3 \%)$ and $0.993( \pm 0.4 \%)$, respectively. In the audit, application of the correction factors improves the mean agreement between treatment plans and measured microDiamond dose from $-2.4 \%( \pm 3.9 \%)$ to $0.4 \%( \pm 3.7 \%)$.

Conclusion: Monte Carlo simulations provide a method for correcting the dose measured in bony materials allowing more accurate comparison with treatment planning system doses. In verification measurements, algorithm specific correction factors should be applied to account for variations in bony material for calculations based on $\mathrm{D}_{\mathrm{m}, \mathrm{m}}$ and $\mathrm{D}_{\mathrm{w}, \mathrm{w}}$.
\end{abstract}

\section{Introduction}

The complexity of treatment delivery has rapidly increased over recent years with a particular shift towards stereotactic treatments including stereotactic body radiation therapy (SBRT) [1-4]. The potential advantages to patients of effective local control with fewer hospital visits can only be realised if these treatments can be delivered safely. In contrast to conventional radiotherapy, stereotactic techniques employ smaller treatment margins and higher doses per fraction, greatly increasing the requirements for accurate dosimetry delivered with precision $[1,5]$. For accurate SBRT treatments the correct vendor supplied tools need to also be underpinned with a strong physics programme.

\footnotetext{
* Corresponding author at: Australian Clinical Dosimetry Service, Australian Radiation Protection and Nuclear Safety Agency, 619 Lower Plenty Rd, Yallambie, VIC, Australia.

E-mail address: maddison.shaw@arpansa.gov.au (M. Shaw).
} 
Technological advances in image-guided radiation therapy, treatment planning and modulated delivery modalities have facilitated safe ablative treatment of extra cranial target volumes with increased conformity and increased geometric precision $[1,5]$. Nonetheless, there remains the weakness that quality assurance of SBRT treatments is commonly performed on homogeneous water or PMMA phantoms [6-8], ignoring the complexities of heterogeneities where dosimetry issues are likely to occur. Improvements in modern phantom materials including synthetic bone mean that suitable heterogeneous phantoms are now readily available $[9,10]$. However, measurements in non-water material are challenging as detector calibrations are only provided for dose to water. This work seeks to fill this gap by providing the modelled corrections for measuring in bone, facilitating improved end-to-end testing on more realistic heterogeneous phantoms for local hospital commissioning and dosimetry audit programmes.

The spine is the most common site of skeletal metastases, affecting almost $40 \%$ of all cancer patients who develop metastatic disease [11-13]. SBRT offers effective local control in the case of spine metastases, making it the treatment of choice for many patients with this disease $[5,13,14]$. SBRT spine treatment plans use complex delivery systems such as intensity-modulated radiotherapy (IMRT), volumetric modulated arc therapy (VMAT) or robotic radiosurgery (Cyberknife). End-to-end testing and dosimetry audits become increasingly important to ensure systemic errors are not present in the patient delivery pathway. The SBRT spine dosimetry audit by Imaging and Radiation Oncology Core (IROC) [9] found $60 \%$ of audit failures were due to systematic dose errors, highlighting the need for accurate dosimetry in end-to-end testing. Previously described SBRT dosimetry audits have addressed the need for non-water material corrections in the measurement of dose in bone. In the end-to-end testing for Spine SBRT described by Hardcastle et.al [15], the authors chose to avoid the bony materials all together and perform measurements in homogenous phantoms, as they lacked correction factors that were required for film measurements in bony regions. In the study by Lee et al. [16], measurements of SBRT spine were $3.9-5.3 \%$ higher than the planned dose when measuring in bony materials, with the discrepancies attributed to non-water like materials and calibration of detectors. Traditionally, radiotherapy detectors are calibrated using a normalised dose to water approach, in a water phantom. Currently no primary standards dosimetry laboratories offer calibrations in non-water media such as bone. Even if a treatment planning system (TPS) calculates dose to water, a measurement in a bone phantom with a detector calibrated in a water phantom will not give the correct answer, as the secondary electrons from the surrounding bone increase the dose compared to calibration conditions in water. In this paper, we measure and calculate the corrections required to directly measure in bony materials and allow comparison to TPS algorithms that calculate either dose to water, in water $\left(D_{\mathrm{w}, \mathrm{w}}\right)$ or dose to medium, in medium $\left(D_{m, m}\right)$.

To calculate the corrections required, the definitions of dose to water and dose to medium firstly need to be clearly defined. Modern TPS used in radiotherapy compute dose to a patient using a variety of dose computation algorithms and mathematical approximations, leading to inconsistencies in the clinical data. TPS have historically calculated radiotherapy dose as dose to water, inwater with variable electron density $\left(D_{w, w}\right)$. This method accounts for radiation transport through differing densities of patient tissues but not the tissue types themselves [17]. In the $D_{w, w}$ case where bony materials are present, the TPS treats these regions as 'high density water', where the material type is water, with a density of that of the bone. Since the introduction of Monte Carlo and Linear Boltzmann Solver based calculations, dose can be reported as dose to medium-in-medium $\left(D_{m, m}\right)$ or as dose to water-in-medium $\left(D_{w}\right.$, $m$ ). For $D_{m, m}$ calculations, the TPS accounts for radiation transport through a number of patient like materials and the density of such materials. For $D_{w, m}$ calculations, the TPS first performs a $D_{m, m}$ calculation, and then converts the dose to $D_{w, m}$ via stopping power ratios for Monte Carlo algorithms [18,19]. For Linear Boltzmann Solver algorithms, such as AcurosXB [20], calculations of electron fluence are performed in patient like materials, regardless of whether $D_{m, m}$ or $D_{w, m}$ is selected. For the final calculation step, the TPS uses medium or water cross sections and densities depending on the reporting quantity. For patient like materials with atomic numbers close to water these processes result in very similar calculations of dose [18]. Large discrepancies of approximately $10 \%$ can be observed between the different reporting methods in regions of high density, such as cortical bone [17-19,21]. Andreo [18] recommended avoiding the conversion from $D_{m, m}$ to $D_{w, m}$ via stopping power ratios due to the uncertainties involved. Gladstone et.al. [22] also recommend reporting $D_{m, m}$ for consistency in NRG clinical trials. For the purposes of this study, the $D_{w}$, $\mathrm{m}$ reporting mode has not been investigated, as all AXB and MC plans included in the analysis were reported as $\mathrm{D}_{\mathrm{m}, \mathrm{m}}$. Absorbed dose is traditionally defined as $\mathrm{D}_{\mathrm{w}, \mathrm{w}}$ [23] and radiotherapy detectors are traditionally calibrated in terms of absorbed dose to water $\left(D_{w, w}\right)$ which creates challenges in dose verification for calculations in bony material. With the aim of a consistent definition of dose, and in particular, only one definition of absorbed dose to water, we propose a methodology for measuring dose in bone as either as $\mathrm{D}_{\mathrm{m}, \mathrm{m}}$ or $\mathrm{D}_{\mathrm{w}, \mathrm{w}}$, based on the primary reporting mode of the calculation algorithm [24]. The methodology is applied in the context of an end-to-end clinical dosimetry audit of SBRT spine treatments. Independent dosimetry audits are recommended by international SBRT guidelines for validation of treatment delivery of such complex techniques $[1,2,25]$. The Australian Clinical Dosimetry Service (ACDS) [26] conducts independent dosimetry audits across Australian and New Zealand Radiation Oncology facilities. The ACDS introduced the SBRT modality into the Level III end-to-end audit program in 2018. The Level III audit is a dosimetric intercomparison where an anthropomorphic phantom undergoes all steps within the patient treatment pathway [27]. The ACDS SBRT audit consists of multiple cases replicating the most common tumour sites treated with SBRT; lung, spine and soft tissue. Measurements are conducted on-site at each facility by ACDS representatives, using an anthropomorphic thorax phantom (CIRS, Norfolk, VA, USA), which includes trabecular and cortical bone, inhale lung and soft tissue equivalent materials. The primary detectors used in the audit are Gafchromic EBT3 radiochromic film (Ashland, Bridgwater, NJ USA) and a PTW 60019 microDiamond (PTW Freiburg, Germany). This paper discusses the measurement of dose in the SBRT spine treatment case of the audit with the application of Monte Carlo modelled correction factors for the bony materials in the phantom, including 63 measured SBRT treatment plans from six treatment planning systems.

\section{Materials and methods}

\subsection{Theoretical considerations}

Discrepancies between the measured and planned doses in the bony regions of the spine were expected due to the detectors being calibrated in terms of absorbed dose to water. This was independent of the algorithm calculating either dose to medium $\left(D_{m, m}\right)$ or dose to water $\left(D_{w, w}\right)$. In the $\mathrm{D}_{\mathrm{m}, \mathrm{m}}$ case, the plan is calculating the dose to bone, but the detector is calibrated in terms of dose to water and so requires a correction. In the $D_{w, w}$ case where bone is present, the planning system is calculating dose to high density water. Whilst the plan is calculating dose to water, the detector in the phantom is surrounded by high $\mathrm{Z}$ bone material which does not match the calibration conditions of the detector measuring in a water phantom. To account for these discrepancies, we propose the use of correction factors $\left(\mathrm{k}_{\mathrm{med}}\right)$ for both the film and microDiamond detectors when measuring in the different bony material as per Eqs. (1) and (2).

$D_{b, b}=M_{b} \quad N_{D, w} \quad k_{\text {med_Db,b }}$

$\mathrm{D}_{\mathrm{HDw}, \mathrm{w}}=\mathrm{M}_{\mathrm{b}} \mathrm{N}_{\mathrm{D}, \mathrm{w}} \mathrm{k}_{\text {med_DHDw,w }}$ 
In the $\mathrm{D}_{\mathrm{m}, \mathrm{m}}$ algorithm scenario, the quantity of interest is the dose in the bony material $\left(\mathrm{D}_{\mathrm{b}, \mathrm{b}}\right)$ which is calculated through measurement in bone $\left(\mathrm{M}_{\mathrm{b}}\right)$, the detector calibration factor $\left(\mathrm{N}_{\mathrm{D}, \mathrm{w}}\right)$ and a medium dependent correction factor $\left(\mathrm{k}_{\mathrm{med}}\right)$. A schematic of modelled correction factors for the $D_{m, m}$ scenario, as measured from a detector in bone $\left(D_{\text {det }, \mathrm{b}}\right)$ to the dose to bone in bone $\left(D_{b, b}\right)$, is shown in Fig. 1. Four situations are modelled for the $\mathrm{k}_{\text {med_Db,b }}$ correction factors. Fig. $1 \mathrm{a}$ shows the definition of what the detector is calibrated to; ie calibration to absorbed dose to water, in a homogeneous water phantom. Fig. 1b then shows the detector calibration conditions; where the detector is in a homogeneous water phantom. The audit measurement conditions are shown in Fig. 1c, with the detector measuring in bone material. Finally, Fig. $1 \mathrm{~d}$ shows the TPS calculation of the dose to a voxel of bone, surrounded by bony material. The dose at the centre voxel in each scenario is used to calculate the $\mathrm{k}_{\text {med }}$ as per Eq. (3).

$k_{\text {med_Db,b }}=\frac{D_{b, b}}{D_{w, w}} \frac{D_{\text {det, } w}}{D_{\text {det, }, \mathrm{b}}}$

For the $\mathrm{D}_{\mathrm{w}, \mathrm{w}}$ algorithm scenario, the quantity of interest is the dose in the bony region, which is treated as 'high density water' by conventional TPS. The correction for conventional TPS calculations using water materials with the density of bone $\left(\mathrm{D}_{\mathrm{HDw}, \mathrm{w}}\right)$ is achieved by replacing the bone in the final schematic Fig. 1(d) with high density water. The proposed $k_{\text {med }}$ for the $D_{w, w}$ scenario is given in Eq. (4).

$\mathrm{k}_{\text {med_DHDw }, \mathrm{w}}=\frac{\mathrm{D}_{\mathrm{HDw}, \mathrm{w}}}{\mathrm{D}_{\mathrm{w}, \mathrm{w}}} \frac{\mathrm{D}_{\text {det }, \mathrm{w}}}{\mathrm{D}_{\text {det }, \mathrm{b}}}$

\subsection{Monte Carlo modelling}

Monte Carlo simulations were performed for Gafchromic Film and stylised microDiamond geometries obtained from the manufacturers' specifications $[28,29]$. All Monte Carlo modelling was performed in the EGSnrc [30] user code DOSXYZnrc [31] with the 2020 version. The source used in these simulations was a phase space file generated in BEAMnrc [32] for $4 \times 4 \mathrm{~cm}^{2}$ fields from a previously validated $6 \mathrm{MV}$ and 10MV Elekta Synergy Linear Accelerator model [33]. A $4 \times 4 \mathrm{~cm}^{2}$ field represents typical field sizes for the SBRT spine plans included in the audit. In all simulations, the incident beam was from above the modelled geometries at $80 \mathrm{~cm}$ SSD. Density correction files generated with the ESTAR [34] program were used for CIRS bony materials and diamond material. All default transport options were used, unless specified otherwise in Table 1. In all cases, the unit being scored is dose, and the uncertainties represent one standard deviation uncertainties calculated with history-by-history statistical analysis. All simulations were performed on the National Computational Infrastructure (NCI) high powered computer cluster with Xeon Platinum 8274 CPUs, and the total computation time to generate the various $\mathrm{k}_{\text {med }}$ factors took between 310 and $1680 \mathrm{~h}$.

EBT3 Gafchromic film consists of $28 \mu \mathrm{m}$ active layer, between two polystyrene layers of $125 \mu \mathrm{m}$ [28]. This structure was modelled parallel
Table 1

EGSnrc Monte Carlo simulation transport settings.

\begin{tabular}{ll}
\hline Transport Setting & Option \\
\hline Global electron cut off energy (ECUT) & $521 \mathrm{keV}$ \\
Global photon cut off energy (PCUT) & $10 \mathrm{keV}$ \\
Boundary crossing algorithm & EXACT \\
Global SMAX & $1 \mathrm{E} 10$ \\
Skin depth for boundary (MFP) & 3 \\
Bremsstrahlung angular sampling & $\mathrm{KM}$ \\
Photon cross sections & $\mathrm{si}$ \\
Bremsstrahlung cross sections & $\mathrm{BH}$ \\
\hline
\end{tabular}

to the incident beam, in the axial plane in the centre of a $25 \mathrm{~mm}$ bone cube. The bone cube consisted of a $15 \mathrm{~mm}$ cube of CIRS Trabecular Bone (density $1.197 \mathrm{~g} / \mathrm{cm}^{3}$ ) surrounded by $5 \mathrm{~mm}$ outer shell of CIRS Cortical Bone (density $1.91 \mathrm{~g} / \mathrm{cm}^{3}$ ). This geometry is a simplistic representation of the body of the phantom vertebrae. The film and bone cube were placed at the centre of a $30 \times 30 \times 30 \mathrm{~cm}^{3}$ cube of water. The resolution of the central bone cube was modelled in $1.0 \mathrm{~mm}^{3}$ voxels to evaluate the interface effects, with the surrounding water voxels at $13.75 \mathrm{~cm}^{3}$. The density and material of the bone cubes were varied to obtain each of the scenarios detailed in Eqs. (3) and (4), for both 6MV and 10MV.

The microDiamond was modelled based on manufacturer provided specifications [29], with the stem of the detector as a $6.9 \times 6.9 \times 40 \mathrm{~mm}$ cuboid of RW3 (polystyrene521icru, density $1.045 \mathrm{~g} / \mathrm{cm}^{3}$ ), and the active diamond layer as a $3.1 \times 3.1 \times 0.001 \mathrm{~mm}$ cuboid of Carbon (density $3.53 \mathrm{~g} / \mathrm{cm}^{3}$ ), located $1 \mathrm{~mm}$ from the end of the stem. Surrounding the microDiamond was a $20 \times 20 \mathrm{~mm}^{3}$ of CIRS trabecular bone, replicating the measurement point of the detector in the SBRT phantom. The microDiamond and bone cube were placed at the centre of a $30 \times 30 \times 30 \mathrm{~cm}^{3}$ cube of water. The density and material of the bone cubes were varied to obtain each of the scenarios detailed in Eqs. (3) and (4) for both 6MV and 10MV. This is a somewhat simplified representation of the microDiamond, which may contribute additional uncertainty to the modelling.

Based on manufacturer supplied material composition data [35], density correction files for the CIRS cortical and trabecular bone were created using the ESTAR program [34]. These density correction files were used to generate the PEGS4 electron stopping power data files for the simulations. For $\mathrm{D}_{\mathrm{m}, \mathrm{m}}$ simulations, the mass density of the CIRS cortical and trabecular bones was $1.91 \mathrm{~g} / \mathrm{cm}^{3}$ and $1.197 \mathrm{~g} / \mathrm{cm}^{3}$ respectively. For $\mathrm{D}_{\mathrm{HD}, \mathrm{w}}$ calculations, (where the voxels are simulated as water with a density of bone), the mass density of water was defined as 1.769 $\mathrm{g} / \mathrm{cm}^{3}$ and $1.156 \mathrm{~g} / \mathrm{cm}^{3}$ respectively for CIRS cortical and trabecular bone respectively, in order to obtain equivalent electron densities [35].

\subsection{SBRT spine dosimetry audit}

The ACDS SBRT audit is performed on a customised CIRS Thorax phantom. The phantom consists of a plastic water body, inhale lung material and a spine composed of CIRS Trabecular Bone and CIRS
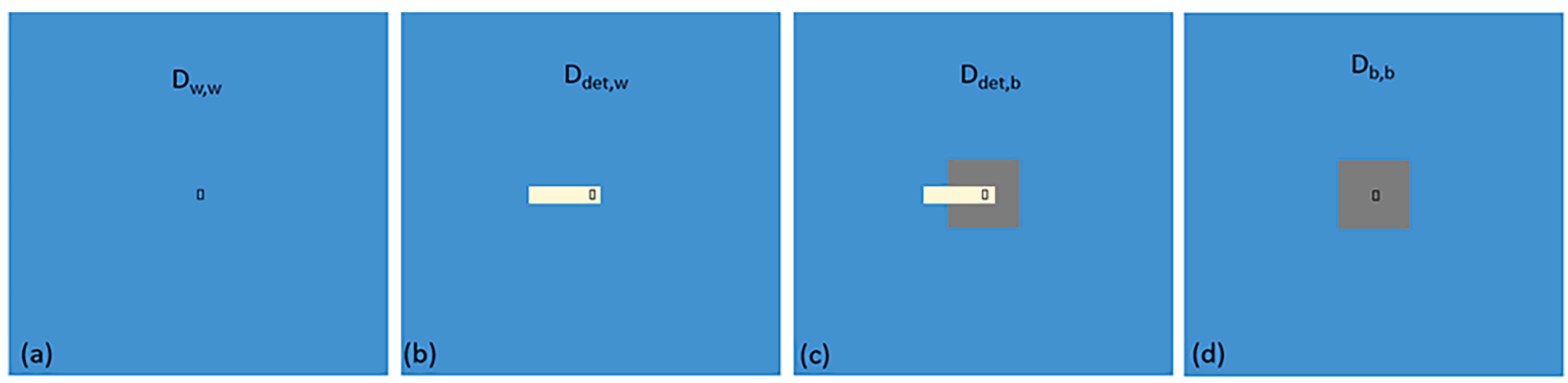

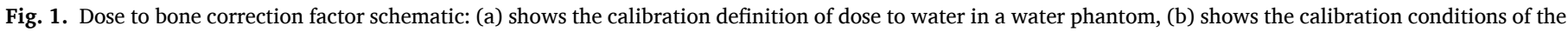
detector in water, (c) shows the measurement conditions of the detector in bone, and (d) shows the TPS calculated dose to medium (bone). 
Cortical Bone [36] (Fig. 2). Weeks prior to the audit, the phantom is mailed to a radiation oncology facility where it undergoes a CT scan, treatment planning and quality assurance according to the local SBRT protocol. ACDS staff then attend the site to perform measurements of the plan. Gafchromic EBT3 Film is used to measure dose at the centre of the target volume in the transverse plane, and two PTW 60019 microdiamond detectors are used for point dose measurements in both the target (trabecular bone) and the spinal cord. Both detectors are calibrated for absolute dosimetry using a normalised dose to water approach $\left(D_{\mathrm{w}, \mathrm{w}}\right)$ at the Australian Primary Standards Laboratory.

Included in the measured film plane are both cortical bone and trabecular bone materials. Using the modelled film correction factors for both cortical and trabecular bone, a correction factor 'spine mask' for both $D_{m, m}$ and $D_{w, w}$ was generated based on the geometry of the anthropomorphic phantom. The resolution of actual CT images of patients as well as the audit phantom leads to blurring in the voxels at the interface of the materials. This is handled in the audit process through the application of a film mask which averages each voxel by the correction factors in the surrounding $3 \mathrm{~mm}$ voxels. The resolution of the film mask was $72 \mathrm{dpi}$, corresponding to pixel size of $0.3 \times 0.3 \mathrm{~cm}^{2}$. The $\mathrm{D}_{\mathrm{m}, \mathrm{m}}$ or $\mathrm{D}_{\mathrm{w}, \mathrm{w}}$ correction factor spine mask was applied to the films measured in the audit, as per the primary reporting mode of the TPS algorithm used in the plan. For analysis of ACDS SBRT audit plans, the measured film is localised to a physical position in the phantom via the facility CT scan. Spatial accuracy of the delivered plan is then assessed using metrics such as distance-to-agreement between planned and measured isodoses and gamma criteria. For this study, the primary objective is to show the difference in planned vs. measured dose due to the bony material. As such, for the purposes of this study only, the measured film and the plan were aligned for best fit to eliminate the spatial inaccuracies in the delivery. The primary metric reported for the purposes of this study is absolute local dose difference between the plan and the measurement. Dose differences were analysed in the PTW Versioft software v6.1. For the microDiamond point dose measurements, the modelled $\mathrm{k}_{\mathrm{med}}$ correction factors were applied to the measured data and compared to the point dose within the plan.

To date, 63 SBRT spine treatment plans have been measured in the audit. Plans were submitted from six TPS; Eclipse (Varian Medical Systems, Palo Alto, CA, USA), Monaco (Elekta AB, Stockholm, Sweden), Pinnacle (Philips Radiation Oncology Systems, Milpitas, CA, USA), RayStation (RaySearch Laboratories, Stockholm, Sweden), iPlan (Brainlab AG, Munich, Germany) and Precision (Accuray, Sunnyvale CA, USA). Table 2 lists the number of plans per algorithm that were included in the SBRT audit, and the calculation method employed. Of the audited plans, $90 \%$ were completed with $6 \mathrm{MV}$ or $6 \mathrm{FFF}$.

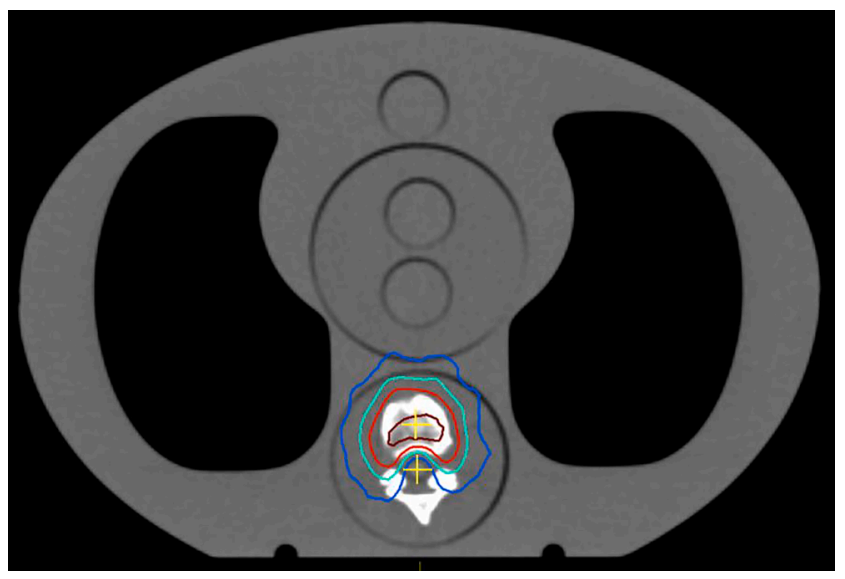

Fig. 2. Transverse CT slice of the ACDS thorax phantom, with trabecular and cortical bone spine, and microdiamond measurement points in spine and spinal cord. A typical planned dose distribution in the film plane is shown.
Table 2

SBRT spine audit plans included in the study.

\begin{tabular}{|c|c|c|c|}
\hline Calculation type & Algorithm & $\begin{array}{l}\text { Treatment } \\
\text { Planning System }\end{array}$ & $\begin{array}{l}\text { No. SBRT } \\
\text { Spine plans }\end{array}$ \\
\hline \multirow{6}{*}{$\begin{array}{l}\mathbf{D}_{\mathrm{m}, \mathrm{m}} \text { Dose to } \\
\text { medium, in } \\
\text { medium }\end{array}$} & AcurosXB (AXB) & Eclipse & 20 \\
\hline & Monte Carlo (MC) & Monaco & 7 \\
\hline & & iPlan & 4 \\
\hline & Collapsed Cone & Pinnacle & 11 \\
\hline & Convolution (CCC)* & RayStation & 2 \\
\hline & $\begin{array}{l}\text { Adaptive Convolve } \\
\text { (AC) }\end{array}$ & Pinnacle & 2 \\
\hline \multirow[t]{3}{*}{$\begin{array}{l}\mathbf{D}_{\mathrm{w}, \mathrm{w}} \text { Dose to water, } \\
\text { in water }\end{array}$} & $\begin{array}{l}\text { Ansiotropic Analytical } \\
\text { Algorithm (AAA) }\end{array}$ & Eclipse & 13 \\
\hline & Ray Tracing (RT) & $\begin{array}{l}\text { Cyberknife } \\
\text { Precision }\end{array}$ & 2 \\
\hline & Monte Carlo (MC) & $\begin{array}{l}\text { Cyberknife } \\
\text { Precision }\end{array}$ & 2 \\
\hline
\end{tabular}

*CCC is dependent on specific implementation of algorithm, and in this work is investigated as both a $\mathrm{D}_{\mathrm{m}, \mathrm{m}}$ and $\mathrm{D}_{\mathrm{w}, \mathrm{w}}$

The Collapsed Cone Convolution (CCC) algorithm implemented in Pinnacle and RayStation TPS, and the Adaptive Convolve (AC) algorithm implemented in Pinnacle TPS are challenging to categorize as they report a mix of $D_{m, m}$ and $D_{w, w}$. These algorithms partially account for material of the voxels by applying material specific mass attenuation coefficients for photon attenuation, which adjusts the water based kernel. Due to the lack of clarity in the literature $[21,22,24,37,38]$, these algorithms have been analysed using both $\mathrm{D}_{\mathrm{m}, \mathrm{m}}$ and $\mathrm{D}_{\mathrm{w}, \mathrm{w}}$.

As the literature recommends avoiding the stopping power/energy deposition ratio based conversion of dose to water in a medium $\left(D_{w, m}\right)$ $[17,18,22,24]$, we have not modelled correction factors for this methodology. Additionally, no SBRT spine plans were submitted to this study using this calculation method.

\section{Results}

\subsection{Material validation}

Validation of the PEGS4 data for the CIRS cortical and trabecular bone materials was performed by comparing transmission measurements and TPS calculations with Monte Carlo modelled results. A CC13 ionisation chamber (IBA Dosimetry, Schwarzenbruck, Germany) and a PTW microDiamond were used to measure the dose from the $6 \mathrm{MV}$ Elekta Synergy $4 \times 4 \mathrm{~cm}^{2}$ field, at $15 \mathrm{~cm}$ depth in a $30 \times 30 \times 30 \mathrm{~cm}^{3}$ CIRS solid water phantom. Measurements were repeated with $15 \times 15$ $\times 2 \mathrm{~cm}^{3}$ slabs of CIRS cortical and trabecular bone to a maximum thickness of $8 \mathrm{~cm}$ placed on top of the water phantom. All measurement scenarios were replicated in the Monaco TPS, using the Monte Carlo algorithm, $\mathrm{D}_{\mathrm{m}, \mathrm{m}}$ reporting mode and a combined statistical uncertainty of $0.5 \%$ per plan. The measurement scenarios were also replicated in simple Monte Carlo models using the EGSnrc user code DOSXYZnrc. Table 3 shows the difference in transmission ratios for the water phantom vs. water phantom with $2 \mathrm{~cm}$ slab of cortical/trabecular bone. The transmission ratio in the EGSnrc monte carlo simulations has been compared to calculations in Monaco TPS (Monte Carlo algorithm) and measurements with $\mathrm{CC} 13$ and microDiamond detectors. For cortical bone, the ratio of the dose in the water and water/bone models for

Table 3

The difference in the transmission dose ratios in water phantom, vs water phantom with $2 \mathrm{~cm}$ cortical and trabecular bone slabs.

\begin{tabular}{|c|c|c|c|}
\hline & \multicolumn{2}{|c|}{$\begin{array}{l}\text { Monte Carlo vs. } \\
\text { Measurement }\end{array}$} & \multirow[t]{2}{*}{ Monte Carlo vs. Monaco MC $\left(\mathrm{D}_{\mathrm{m}, \mathrm{m}}\right)$} \\
\hline & $\mathrm{CC} 13$ & microDiamond & \\
\hline Trabecular bone & $0.5 \%$ & $0.6 \%$ & $0.6 \%$ \\
\hline Cortical bone & $-0.5 \%$ & $-0.3 \%$ & $0.7 \%$ \\
\hline
\end{tabular}


thickness of cortical bone ranging from $2 \mathrm{~cm}$ to $8 \mathrm{~cm}$ was found to be within $1.0 \%$ of CC13 measurements and $0.7 \%$ of microDiamond measurements. For the trabecular bone, measurements were performed using a single $2 \mathrm{~cm}$ bone slab due to availability of equipment. The combined uncertainty in the Monte Carlo models was $0.4 \%$.

\subsection{Algorithm specific corrections}

The 2D dose maps in each of the simulated Gafchromic film scenarios are shown in Fig. 3. $\mathrm{k}_{\text {med }}$ correction factors were calculated for each voxel in the $\mathrm{D}_{\mathrm{m}, \mathrm{m}}$ (Fig. 3a-d) and $\mathrm{D}_{\mathrm{HDw}, \mathrm{w}}$ (Fig. 3f-i) scenario DOSXYZnrc input files as per Eqs. (2) and (4). Calculations were performed for both $6 \mathrm{MV}$ and $10 \mathrm{MV}$ beams. The resulting correction factor maps for $6 \mathrm{MV}$ are shown in Fig. 3e and j. For the Gafchromic Film, the final CIRS cortical and trabecular bone $\mathrm{k}_{\text {med }}$ correction factors were averaged across the 3 $\mathrm{mm}$ voxels at the centre of each bone region, avoiding the voxels at the direct interface. The final correction factors for Gafchromic film are summarised in Table 4. The uncertainty in the MC correction factors only accounts for the statistical uncertainty in the MC calculations. For $\mathrm{D}_{\mathrm{m}, \mathrm{m}}$ scenario, the difference between $6 \mathrm{MV}$ and $10 \mathrm{MV}$ was $<0.2 \%$ for both cortical and trabecular bone. For the $\mathrm{D}_{\mathrm{w}, \mathrm{w}}$ scenario, the difference between $6 \mathrm{MV}$ and $10 \mathrm{MV}$ was $1.1 \%$ and $0.9 \%$ for cortical and trabecular bone respectively.

The results of the simulated Gafchromic film and PTW 60019 microDiamond correction factors are summarised in Table 4. For the microDiamond, the correction factors were determined from the voxels in the active layer and the $1 \mathrm{~mm}$ tip of the detector. The difference for all modelled correction factors for the microDiamond between $6 \mathrm{MV}$ and $10 \mathrm{MV}$ was $<0.2 \%$.

Fig. $4 a$ and $b$ show the sagittal central axis profiles across the Gafchromic film $k_{\text {med }}$ correction factor maps for the $D_{m, m}$ and $D_{w, w}$ scenarios. Sharp changes in dose, and resulting correction factors, are seen at the interface regions between the cortical and trabecular bone, particularly for the $\mathrm{D}_{\mathrm{m}, \mathrm{m}}$ scenario. Smoothed profiles were created to reflect the blurring of interfaces which occurs in clinical and audit plans based on CT resolution. The profiles were smoothed across $3 \mathrm{~mm}$, to show the handling of the blurring in the spine mask.

\subsection{Audit results - Gafchromic EBT3 film}

The audit film measurements were corrected using the spine mask and the $\mathrm{k}_{\text {med }}$ factors listed in Table 4, according to the primary reporting mode of the algorithm used in each plan. The 6MV correction factors were applied to plans using $6 \mathrm{MV}$ and $6 \mathrm{FFF}$, while the 10MV correction factors were applied to plans using 10MV and 10FFF. The average uncorrected and corrected local dose difference maps in the SBRT spine audit are shown per algorithm in Fig. 5 and Fig. 6. Table 5 shows the average local dose difference across the scored area of the film, encompassing both cortical and trabecular bone. Algorithms with fewer than 5 audit plans have been excluded from the analysis. The large dose difference seen in the cortical bone region in the top row is notably improved after the film mask is applied.

Fig. 6 shows the average local dose difference maps for the CCC algorithm; uncorrected, corrected for $\mathrm{D}_{\mathrm{m}, \mathrm{m}}$ and corrected for $\mathrm{D}_{\mathrm{w}, \mathrm{w}}$. The classification of Pinnacle CCC is more complex than other algorithms, as this algorithm reports a mix of $\mathrm{D}_{\mathrm{m}, \mathrm{m}}$ and $\mathrm{D}_{\mathrm{w}, \mathrm{w}}$. However following AAPM Task Group 329 [24] it can be considered $\mathrm{D}_{\mathrm{m}, \mathrm{m}}$ in this context. We have included results from correcting by both dose to medium and dose to water for completeness. The dose to medium correction appears to overcorrect in the cortical bone region, which is also evident in the average local dose differences in Table 5.

\subsection{Audit results - PTW 60019 microDiamond point dose}

Fig. 7 shows the per algorithm results of the PTW 60019 microDiamond point dose in trabecular bone in the spine target volume. The $6 \mathrm{MV}$ correction factors were applied to all measurements, as there was no significant energy dependence shown in the microDiamond correction factor simulations. Algorithms with fewer than 5 audit plans have been excluded from the analysis. The mean and standard deviation for the local dose discrepancy of the uncorrected measurements was $-2.4 \%$ and $3.9 \%$ respectively. Application of the algorithm specific correction factors brings the mean local dose discrepancy to $0.4 \%$, with a standard deviation $3.7 \%$.

\section{Discussion}

With the introduction of modern TPS algorithms, there has been much debate in the literature around the clinical impacts of calculations based on patient like materials. For patient media with atomic numbers close to water, calculations of $D_{m, m}$ and $D_{w, w}$ show only very minor differences. As a large proportion of clinical radiotherapy focuses on

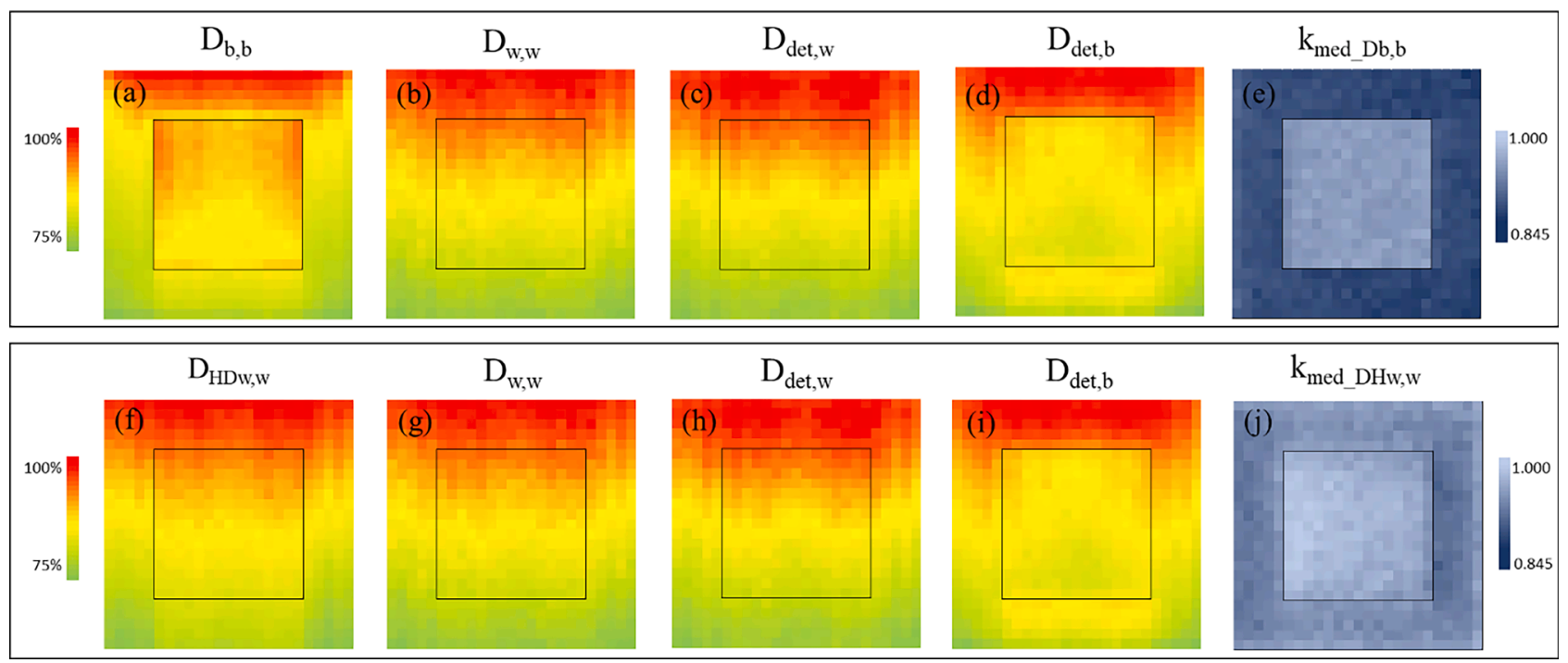

Fig. 3. 2D dose map results of DOSXYZnrc simulations in the central bone cube for the $6 M V$ dose to medium scenario: (a) $D_{b, b}$, (b) $D_{w, w}$, (c) $D_{d e t, w}$ and (d) $D_{\text {det, }, b}$, and the corresponding dose maps for dose to water scenario (f) - (i). In all simulations, the incident beam is from above. The resulting $k_{m e d} 2 \mathrm{D}$ correction factor maps are shown (e) and (j). 
Table 4

Algorithm specific correction factors $\left(\mathrm{k}_{\mathrm{med}}\right)$.

\begin{tabular}{|c|c|c|c|c|c|c|c|}
\hline \multirow[t]{3}{*}{ Calculation type } & \multirow[t]{3}{*}{ Algorithm } & \multicolumn{4}{|c|}{$\mathrm{k}_{\mathrm{med}}$ EBT3 Film } & \multirow{2}{*}{\multicolumn{2}{|c|}{$\begin{array}{l}\mathrm{k}_{\text {med }} \text { PTW } 600019 \\
\text { microDiamond }\end{array}$}} \\
\hline & & \multicolumn{2}{|c|}{ CIRS Cortical bone } & \multicolumn{2}{|c|}{ CIRS Trabecular bone } & & \\
\hline & & $6 \mathrm{MV}$ & $10 \mathrm{MV}$ & $6 \mathrm{MV}$ & $10 \mathrm{MV}$ & $6 \mathrm{MV}$ & $10 \mathrm{MV}$ \\
\hline $\begin{array}{l}\mathrm{D}_{\mathrm{m}, \mathrm{m}} \text { Dose to medium, in } \\
\text { medium }\end{array}$ & $\begin{array}{l}\text { AcurosXB (AXB) } \\
\text { Monte Carlo (MC) } \\
\text { Collapsed Cone Convolution (CCC) } \\
\text { Adaptive Convolve (AC) }\end{array}$ & $\begin{array}{l}0.875 \\
( \pm 0.1 \%)\end{array}$ & $\begin{array}{l}0.876 \\
( \pm 0.1 \%)\end{array}$ & $\begin{array}{l}0.953 \\
( \pm 0.3 \%)\end{array}$ & $\begin{array}{l}0.955 \\
( \pm 0.2 \%)\end{array}$ & $\begin{array}{l}0.962 \\
( \pm 0.4 \%)\end{array}$ & $\begin{array}{l}0.963 \\
( \pm 0.2 \%)\end{array}$ \\
\hline $\mathbf{D}_{\mathbf{w}, \mathbf{w}}$ Dose to water, in water & $\begin{array}{l}\text { Ansiotropic Analytical Algorithm } \\
\text { (AAA) } \\
\text { Ray Tracing (RT) }\end{array}$ & $\begin{array}{l}0.920 \\
( \pm 0.1 \%)\end{array}$ & $\begin{array}{l}0.910 \\
( \pm 0.1 \%)\end{array}$ & $\begin{array}{l}0.982 \\
( \pm 0.3 \%)\end{array}$ & $\begin{array}{l}0.973 \\
( \pm 0.2 \%)\end{array}$ & $\begin{array}{l}0.993 \\
( \pm 0.4 \%)\end{array}$ & $\begin{array}{l}0.994 \\
( \pm 0.2 \%)\end{array}$ \\
\hline
\end{tabular}
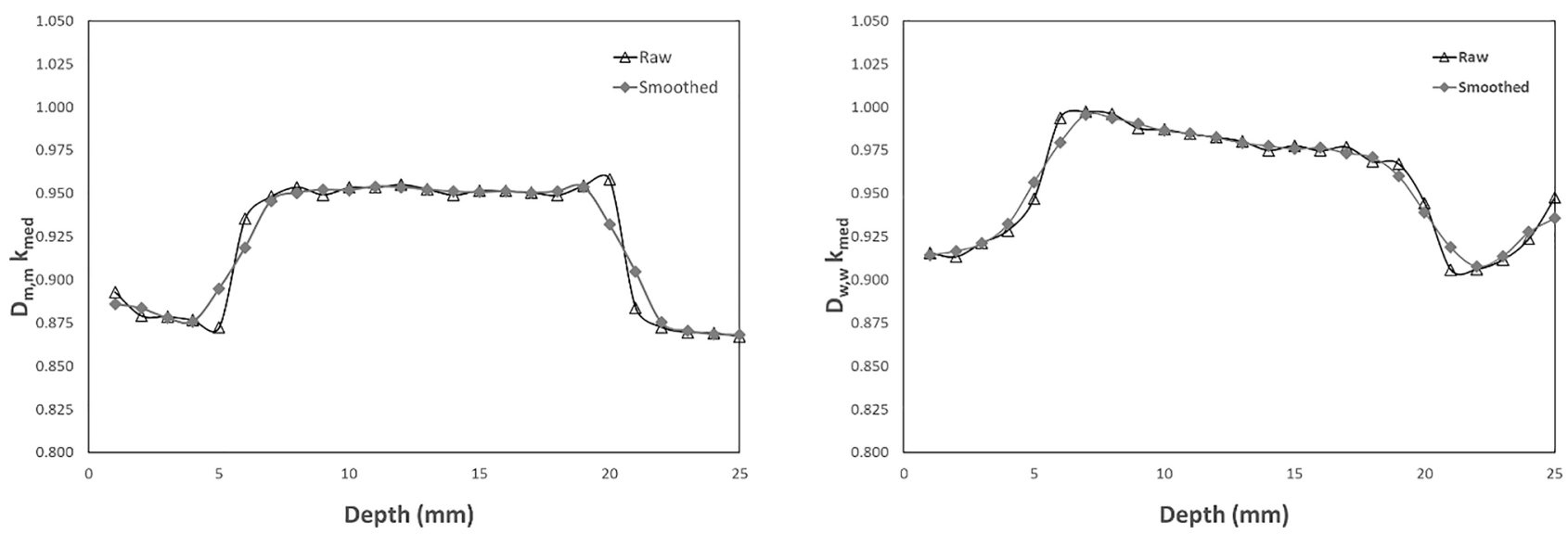

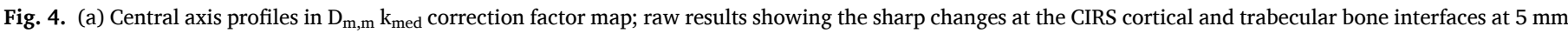

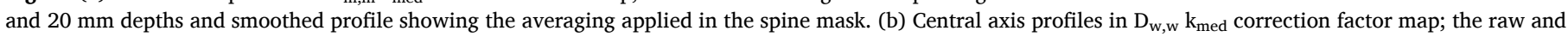
smoothed profiles show better agreement in the high density water scenario.
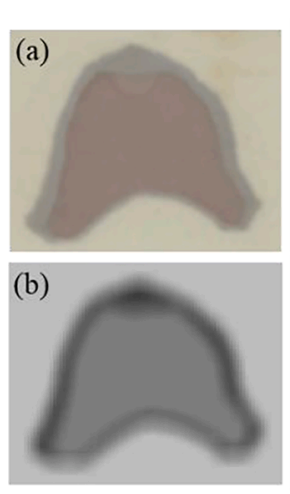
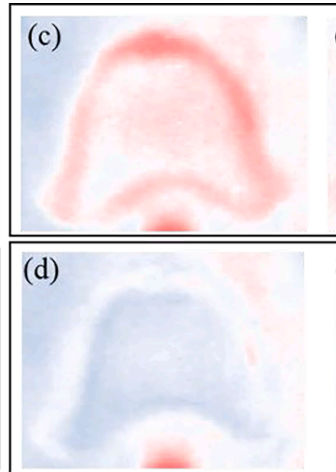

Eclipse AXB (e)

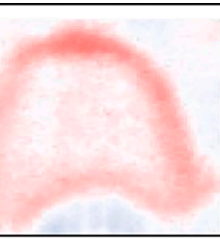

(f) (g)

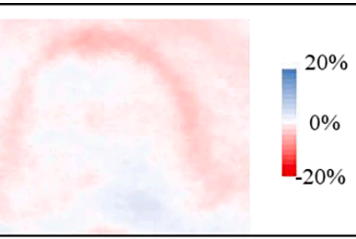

(h)

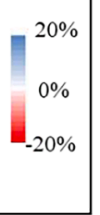

Eclipse AAA
Uncorrected dose difference (film vs plan)

Corrected dose difference (film vs plan)

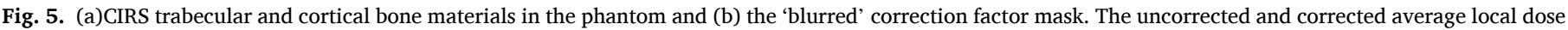
difference maps (film vs. planned dose) of the SBRT spine audit for (c,d) Eclipse AXB, (e,f) Monaco MC, (g,h) Eclipse AAA.

\section{Table 5}

Uncorrected and corrected average local dose difference (\%) (film vs. planned dose) across scored area of film for SBRT spine audit (encompassing both cortical and trabecular bone).

\begin{tabular}{llll}
\hline \multirow{2}{*}{ Algorithm } & & \multicolumn{2}{l}{ Average local dose difference (\%) } \\
\cline { 3 - 4 } & Raw & Corrected $\mathrm{D}_{\mathrm{m}, \mathrm{m}}$ & Corrected $\mathrm{D}_{\mathrm{w}, \mathrm{w}}$ \\
\hline Eclipse AXB & $-1.4 \%$ & $1.2 \%$ & \\
Monaco MC & $-1.8 \%$ & $0.7 \%$ & $0.1 \%$ \\
Eclipse AAA & $-1.1 \%$ & & $1.2 \%$ \\
Collapsed Cone & $0.6 \%$ & $3.4 \%$ & \\
\hline
\end{tabular}

treatment of tumours in soft tissue regions, the impact of $\mathrm{D}_{\mathrm{m}, \mathrm{m}}$ calculations will be rather insignificant. However, in patient materials with high density such as cortical bone, the differences between $D_{m, m}$ and $D_{w}$, ${ }_{\mathrm{w}}$ calculations can be up to $10 \%[17-19,21]$. One of the concerns with the $D_{m, m}$ reporting mode is the challenges of verifying dose in non water like media such as bone. Traditional patient specific QA often employs the use of a homogenous phantom for verification of dose delivery. The radiation detectors used for patient specific QA are calibrated dose-towater, which leads to large discrepancies in measured dose for plans with regions of bony material. The Monte Carlo simulations in this paper provide a method for correction of measured dose in bone like materials in an SBRT spine phantom. Whilst the bone like materials included in 

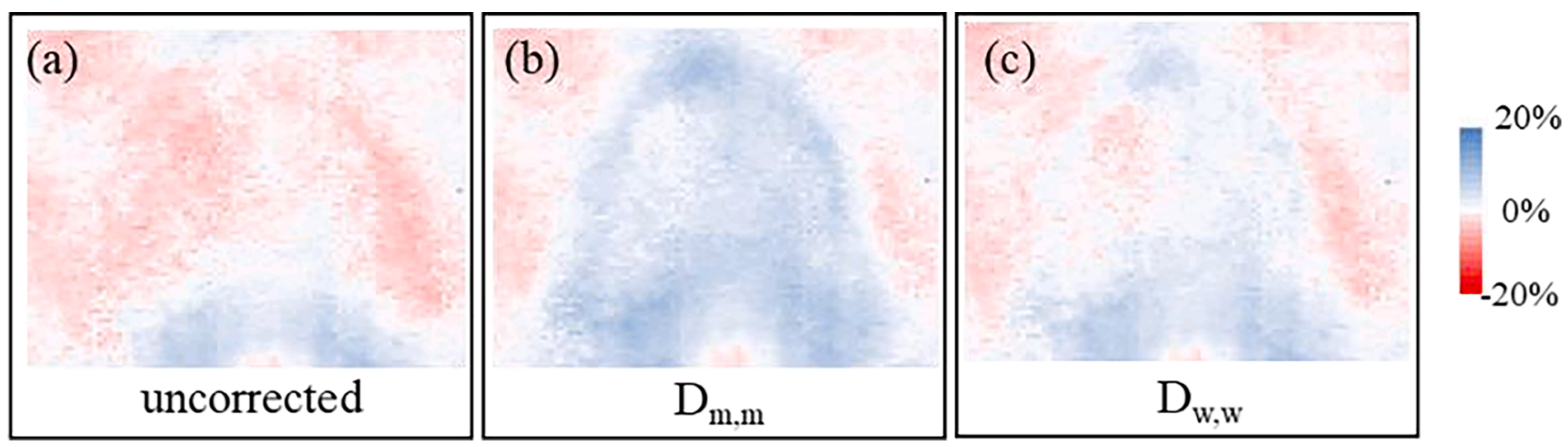

Fig. 6. Average local dose difference maps (film vs. planned dose) for the Pinnacle CCC audit plans; (a) uncorrected, (b) corrected for $\mathrm{D}_{\mathrm{m}, \mathrm{m}}$ and (c) corrected for $\mathrm{D}_{\mathrm{w}, \mathrm{w}}$.

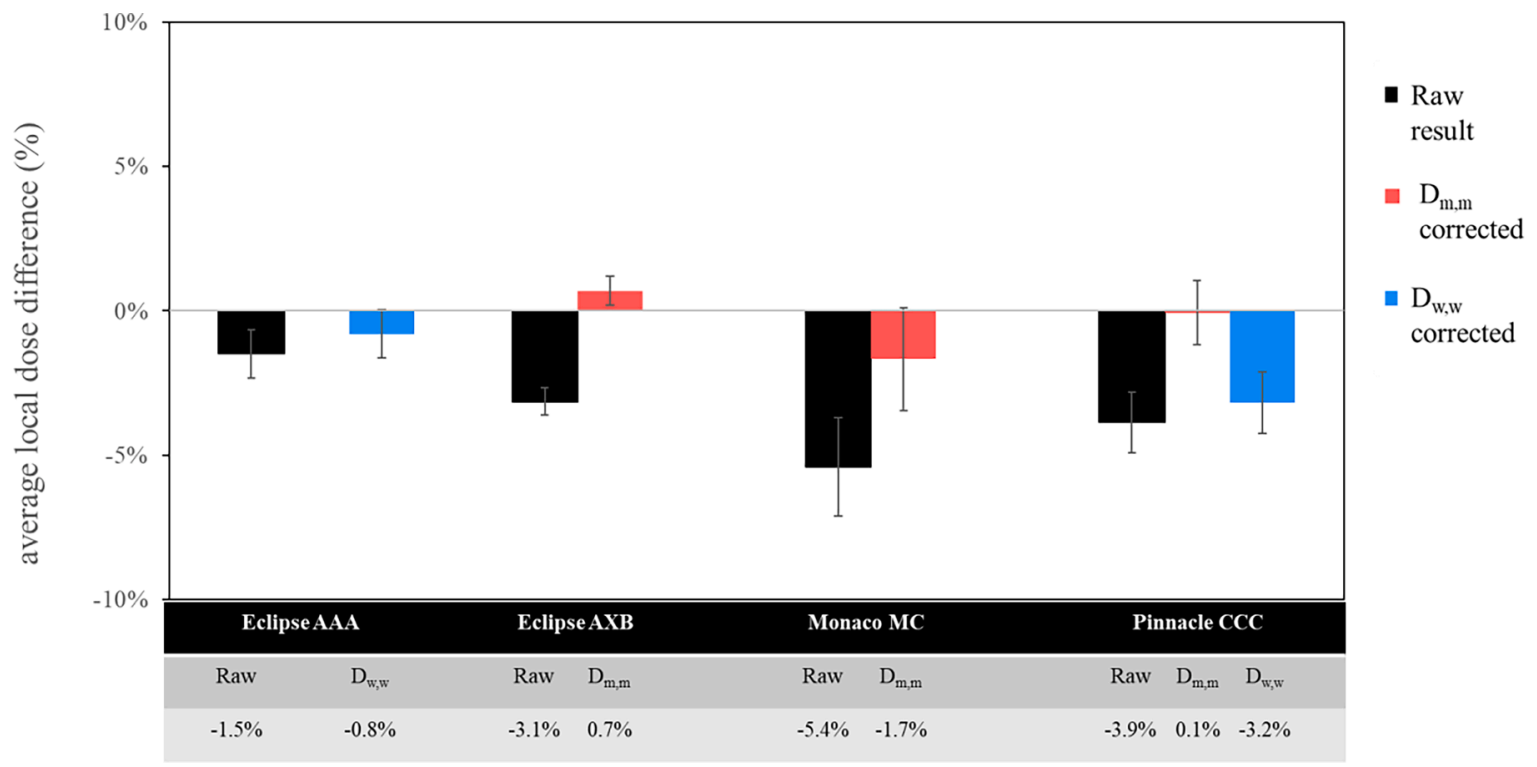

Fig. 7. Average local dose difference in PTW 60019 microDiamond point dose measurements per algorithm. The standard uncertainty in the point dose measurements is shown per algorithm.

this study simulate patient tissue, traceability of non-water materials is not established. According to manufacturer data, the linear attenuations of simulated bone materials in the phantom are within $1 \%$ of actual attenuation in bone [36]. This was also verified by in-house attenuation measurements compared to MC modelled results, however due to the obvious ethical and technical challenges involved, no measurements were done with actual human bone for comparison as would typically be done when commissioning synthetic/plastic water.

The MC corrections for film measurements of treatment plans using $D_{m, m}$ calculations were found to be $4.7 \%$ for trabecular bone and $12.5 \%$ for cortical bone. The corrections for algorithms using $\mathrm{D}_{\mathrm{w}, \mathrm{w}}$ calculations were $1.8 \%$ for trabecular and $8.0 \%$ for cortical bone. When these corrections were applied to the film results from the audit, the large dose differences seen in the cortical bone region were significantly improved. Similar corrections were calculated for the microDiamond measuring in trabecular bone of $0.7 \%$ and $3.8 \%$ for $\mathrm{D}_{\mathrm{w}, \mathrm{w}}$ and $\mathrm{D}_{\mathrm{m}, \mathrm{m}}$ respectively. When the correction was applied to the audit results the overall average dose difference between planned and measured doses improved from $-2.4 \%$ to $0.4 \%$. In this study we have used a simplified microDiamond geometry in the MC simulations. For the SBRT audit, the microDiamond detector for point dose verification is a secondary measurement, with film being the primary detector. The microDiamond local point dose discrepancies show substantial uncertainty, with a standard deviation of $3.7 \%$. This is largely due to the steep gradients seen in the SBRT spine plans. The point dose measurement is designed to offer a coarse check of the plan in real time, with the film serving as the primary detector due to the information it provides on both dosimetric and spatial accuracy of the delivery. The simple model does appear to provide a good approximation for the purposes of a dose in medium correction and shows significant improvement in the average local point dose differences measured in the audit scenario.

A $4 \times 4 \mathrm{~cm}^{2}$ field size was chosen in this study to ensure full coverage of the $2.5 \mathrm{~cm}$ bone cube at the centre of the modelled geometries. A $4 \times$ $4 \mathrm{~cm}^{2}$ field also represents typical field sizes for the SBRT spine plans included in the audit. A limitation of this study is the use of a single field in the Monte Carlo simulations, while the vast majority of the audit plans were delivered with a VMAT technique. The modelled correction factors for bony materials may also be field size dependent; however, both field size and delivery technique differences are outside the scope of the current work, and could be the subject of future investigations. Another limitation of the study was the use of a $6 \mathrm{MV}$ and $10 \mathrm{MV}$ beam for the simulations, whilst many of the audit plans were delivered with flattening filter free beams (FFF). There may be differences in the correction factors for flat beams vs. flattening filter free beams that have not been investigated. In this study we have applied the $6 \mathrm{MV}$ correction factors to $6 \mathrm{FFF}$ treatment plans, and $10 \mathrm{MV}$ to $10 \mathrm{FFF}$ plans. For the $\mathrm{D}_{\mathrm{m}, \mathrm{m}}$ calculation modes, the difference between all correction factors for $6 \mathrm{MV}$ and $10 \mathrm{MV}$ was $<0.2 \%$, within the uncertainty of the Monte Carlo simulations. For the $D_{w, w}$ calculation mode, the difference between the correction factors Gafchromic film for $6 \mathrm{MV}$ and $10 \mathrm{MV}$ was $\sim 1.0 \%$, 
which could contribute additional uncertainty to the results of a 6FFF or $10 \mathrm{FFF}$ audit plan. It is also intriguing that the $\mathrm{D}_{\mathrm{w}, \mathrm{w}}$ simulations showed a difference between the 6MV and 10MV correction factors for film, while the $\mathrm{D}_{\mathrm{m}, \mathrm{m}}$ scenario did not. It is plausible that when the medium is water of variable density, there are more significant differences between the energies. Whilst the differences are relatively small, this concept could be the subject of further investigations. Of note, in the SBRT audit 70\% of the audited spine plans were calculated using the $D_{m, m}$ calculation method, and $90 \%$ of the plans were delivered with either $6 \mathrm{MV}$ or $6 \mathrm{FFF}$.

Large corrections are presented for the Gafchromic film measurements, particularly for the regions of cortical bone. Fig. 4a shows a sharp change in $k_{\text {med }}$ profile at the interface regions for $D_{m, m}$ calculations, while the $D_{w, w}$ models in Fig. $4 \mathrm{~b}$ show a much softer change. These results are similar to the results of the study by Han et.al [21], where MC simulations and the AXB algorithm predicted the peaks and troughs at bone interfaces, whereas AAA did not. Bone interfaces will have large impacts on the final results of the film measurements if some blurring is not employed in the application of the spine mask. This was handled by averaging each voxel in the spine mask by the surrounding $3 \mathrm{~mm}$ voxels. Studies by Reynaert et. al. [17] and Ma [19] showed that interface effects become smoothed out using $2 \mathrm{~mm}$ voxels, a clinically realistic voxel size for SBRT spine plans. The majority of the audit plans included in this study were calculated on a $2 \mathrm{~mm}$ dose calculation grid resolution.

The CCC algorithm as implemented by Pinnacle TPS is complex and falls somewhere between $D_{m, m}$ and $D_{w, w}[21,24]$. For the CCC plans included in the audit, we present both the $\mathrm{D}_{\mathrm{m}, \mathrm{m}}$ and $\mathrm{D}_{\mathrm{w}, \mathrm{w}}$ corrections for the film (Fig. 6) and microDiamond (Fig. 7). The ACDS plans to apply the $\mathrm{D}_{\mathrm{m}, \mathrm{m}} \mathrm{k}_{\mathrm{med}}$ corrections factors to Pinnacle CCC plans included in the audit, as per TG-329 [24]. For the microDiamond measurements, the $\mathrm{D}_{\mathrm{m}, \mathrm{m}}$ corrections appear to be working, taking the average local dose difference from $-3.4 \%$ uncorrected, to $0.4 \%$ corrected. For the film measurements, the $\mathrm{D}_{\mathrm{m}, \mathrm{m}}$ does appear to be somewhat overcorrecting as seen by the average local dose difference of $3.4 \%$ in Table 5 and in Fig. 6c.

The audit data shows that a number of different algorithm and reporting mode approaches are currently in used in modern radiotherapy. Comparing plans in large scale analysis projects such as SBRT spine clinical trial credentialing is difficult if differences in measurement are not addressed first. The results of this paper show the importance of addressing these differences when comparing a diverse cohort of data. The Monte Carlo simulations performed show large corrections for measurement of dose in cortical and trabecular bone. Dosimetric verification of SBRT spine plans often involve the use of Gafchromic Film for both dosimetric and spatial accuracy. Absolute dose difference and gamma criteria are common metrics used for analysis of patient specific QA measurements. Excluding a medium correction in regions of the patient plan with bony material may have significant impacts on absolute dose differences and gamma passing rates due to the dose discrepancies in bone. The impacts of the medium correction are also relevant for plans other than SBRT spine. Similar discrepancies will be seen in plans which have regions of dense bone are included in the calculation, such as treatments involving the skull. Consideration should be given to verification measurements where dense bone is present in the target volumes, and/or organs at risk. For end to end testing and PSQA where the phantom include regions of bony like material, we recommend calculation of algorithm specific corrections for radiotherapy detectors to improve the accuracy of measurement

\section{Conclusion}

Monte Carlo simulations provide a method for correcting the dose measured in bone like materials in a SBRT spine phantom to allow accurate comparison with treatment planning system doses. The ACDS has adopted this methodology in the end-to-end SBRT spine audit, where the overall local dose difference between planned and measurements decreases from $-2.4 \%$ to $0.4 \%$. To improve accuracy of verification measurements, algorithm specific correction factors should be applied to account for variations in bony material for calculations based on $D_{m, m}$ and $\mathrm{D}_{\mathrm{w}, \mathrm{w}}$.

\section{Acknowledgements}

This research is supported by an Australian Government Research Training Program (RTP) Scholarship.

The Monte Carlo component of this research was undertaken with the assistance of resources and services from the National Computational Infrastructure (NCI), which is supported by the Australian Government.

\section{References}

[1] Foote M, Bailey M, Smith L, Siva S, Hegi-Johnson F, Seeley A, et al. Guidelines for safe practice of stereotactic body (ablative) radiation therapy. J Med Imaging Radiat Oncol 2015;59:646-53.

[2] Sahgal A, Roberge D, Schellenberg D, Purdie T, Swaminath A, Pantarotto J, et al. The Canadian Association of Radiation Oncology scope of practice guidelines for lung, liver and spine stereotactic body radiotherapy. Clin Oncol 2012;24:629-39.

[3] Distefano G, Baker A, Scott A, Webster G. Survey of stereotactic ablative body radiotherapy in the UK by the QA group on behalf of the UK SABR Consortium. Br J Radiol 2014;87:20130681.

[4] Pan H, Simpson DR, Mell LK, Mundt AJ, Lawson JD. A survey of stereotactic body radiotherapy use in the United States. Cancer 2011;117:4566-72.

[5] Zeng KL, Tseng C-L, Soliman H, Weiss Y, Sahgal A, Myrehaug S. Stereotactic body radiotherapy (SBRT) for oligometastatic spine metastases: an overview. Front Oncol 2019;9:337.

[6] Hussein M, Rowshanfarzad P, Ebert MA, Nisbet A, Clark CH. A comparison of the gamma index analysis in various commercial IMRT/VMAT QA systems. Radiother Oncol 2013;109:370-6.

[7] Kim J-i, Park S-Y, Kim HJ, Kim JH, Ye S-J, Park JM. The sensitivity of gamma-index method to the positioning errors of high-definition MLC in patient-specific VMAT QA for SBRT. Radiat Oncol 2014;9:1-12.

[8] Hillman Y, Kim J, Chetty I, Wen N. Refinement of MLC modeling improves commercial QA dosimetry system for SRS and SBRT patient-specific QA. Med Phys 2018;45:1351-9.

[9] Edward SS, Alvarez PE, Taylor PA, Molineu HA, Peterson CB, Followill DS, et al. Differences in the patterns of failure between IROC lung and spine phantom irradiations. Pract Radiat Oncol 2020.

[10] Clark CH, Hurkmans CW, Kry SF, of Radiation TGQA, Group TCTH. The role of dosimetry audit in lung SBRT multi-centre clinical trials. Phys Med 2017.

[11] Chang EL, Shiu AS, Mendel E, Mathews LA, Mahajan A, Allen PK, et al. Phase I/II study of stereotactic body radiotherapy for spinal metastasis and its pattern of failure. J Neurosurg: Spine 2007;7:151-60.

[12] Klimo P, Kestle JR, Schmidt MH. Clinical trials and evidence-based medicine for metastatic spine disease. Neurosurg Clin 2004;15:549-64.

[13] Wang XS, Rhines LD, Shiu AS, Yang JN, Selek U, Gning I, et al. Stereotactic body radiation therapy for management of spinal metastases in patients without spinal cord compression: a phase 1-2 trial. Lancet Oncol 2012;13:395-402.

[14] Tseng C-L, Eppinga W, Charest-Morin R, Soliman H, Myrehaug S, Maralani PJ, et al. Spine stereotactic body radiotherapy: indications, outcomes, and points of caution. Global Spine J 2017;7:179-97.

[15] Hardcastle N, Kron T, Cook O, Lehmann J, Mitchell PL, Siva S. Credentialing of vertebral stereotactic ablative body radiotherapy in a multi-centre trial. Physica Med 2020;72:16-21.

[16] Lee J, Patel R, Eaton D, Clark C. The impact of dose to medium on the results of a national spine SBRT dosimetry audit. Radiotherapy and Oncology: ELSEVIER IRELAND LTD ELSEVIER HOUSE, BROOKVALE PLAZA, EAST PARK SHANNON, CO...; 2019. p. S568-S.

[17] Reynaert N, Crop F, Sterpin E, Kawrakow I, Palmans H. On the conversion of dose to bone to dose to water in radiotherapy treatment planning systems. Phys Imaging Radiat Oncol 2018;5:26-30.

[18] Andreo P. Dose to 'water-like'media or dose to tissue in MV photons radiotherapy treatment planning: still a matter of debate. Phys Med Biol 2014;60:309.

[19] Ma C, Li J. Dose specification for radiation therapy: dose to water or dose to medium? Phys Med Biol 2011;56:3073.

[20] Failla GA, Wareing T, Archambault Y, Thompson S. Acuros XB advanced dose calculation for the Eclipse treatment planning system. Palo Alto, CA: Varian Medical Systems; 2010.

[21] Han T, Mikell JK, Salehpour M, Mourtada F. Dosimetric comparison of Acuros XB deterministic radiation transport method with Monte Carlo and model-based convolution methods in heterogeneous media. Med Phys 2011;38:2651-64.

[22] Gladstone DJ, Kry SF, Xiao Y, Chetty IJ. Dose specification for NRG radiation therapy trials. Int J Radiat Oncol Biol Phys 2016;95:1344-5.

[23] Andreo P, Burns D, Hohlfeld K, Huq MS, Kanai T, Laitano F, et al. Absorbed dose determination in external beam radiotherapy: an international code of practice for dosimetry based on standards of absorbed dose to water. IAEA TRS. 2000;398.

[24] Kry SF, Feygelman V, Balter P, Knöös T, Ma CMC, Snyder M, et al. AAPM Task Group 329: reference dose specification for dose calculations: dose-to-water or dose-to-muscle? Med Phys 2019. 
[25] Kirkbride P, Cooper T. Stereotactic body radiotherapy. Guidelines for commissioners, providers and clinicians: a national report. WB Saunders; 2011.

[26] Williams I, Kenny J, Lye J, Lehmann J, Dunn L, Kron T. The Australian Clinical Dosimetry Service: a commentary on the first 18 months. Australas Phys Eng Sci Med 2012;35:407-11.

[27] Kron T, Hamilton C, Roff M, Denham J. Dosimetric intercomparison for two Australasian clinical trials using an anthropomorphic phantom. Int J Radiat Oncol Biol Phys 2002;52:566-79.

[28] Ashland BNU. GAFCHROMICTM DOSIMETRY MEDIA, TYPE EBT-3. http://www.ga fchromic.com/documents/EBT3_Specifications.pdf.

[29] Germany PF. PTW 60019 microDiamond detector specifications. Private Communication. 2018

[30] Kawrakow I, Mainegra-Hing E, Rogers DWO, Tessier F, Walters BRB. The EGSnrc Code System: Monte Carlo Simulation of Electron and Photon Transport. NRCC Report PIRS-701: Ottawa: National Research Council Canada; 2020.

[31] Walters BRB, Kawrakow I, D.W.O R. DOSXYZnrc Users Manual. NRCC Report PIRS794revB: Ottowa: National Research Council Canada; 2020.
[32] Rogers DWO, Walters BRB, Kawrakow I. BEAMnrc Users Manual. NRCC Report PIRS-0509(A)revL: Ottowa: National Research Council Canada; 2020.

[33] Lye J, Butler D, Ramanathan G, Franich R. Spectral differences in 6 MV beams with matched PDDs and the effect on chamber response. Phys Med Biol 2012;57:7599.

[34] Berger MJ CJ, Zucker MA, Chang J. ESTAR.: Stopping-power and range tables for electrons, protons, and helium ions http://www.nist.gov/pml/ data/star/index. cfm NIST; 2011.

[35] Computerized Imaging Reference Systems I. CIRS Custom Phantom TissueEquivalent Materials Elemental Composition Data and Attenuation Coefficients, Project 1633-01. In: Service ACD, editor.December 15, 2017.

[36] Technology CTSaP. E2E SBRT Phantom Model 036A-CV-XX-xx. 036A-CVXX-xx DS 070716. www.cirsinc.com 2013.

[37] Ahnesjö A. Collapsed cone convolution of radiant energy for photon dose calculation in heterogeneous media. Med Phys 1989;16:577-92.

[38] Fotina I, Winkler P, Künzler T, Reiterer J, Simmat I, Georg D. Advanced kernel methods vs. Monte Carlo-based dose calculation for high energy photon beams. Radiother Oncol 2009;93:645-53. 